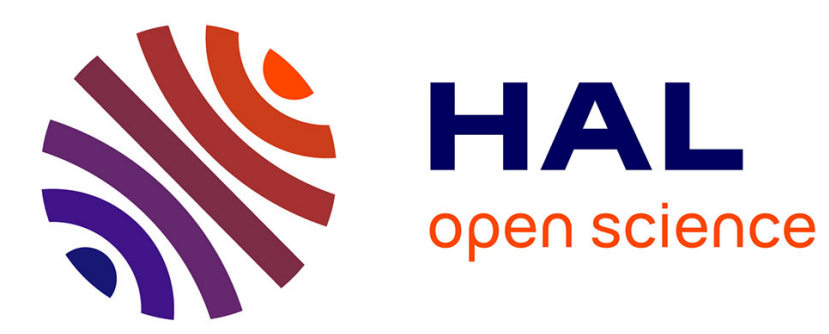

\title{
On robust stability of multistable passive systems
} Nelson de Figueireido Barroso, Rosane Ushirobira, Denis Efimov, Alexandre L Fradkov

\section{To cite this version:}

Nelson de Figueireido Barroso, Rosane Ushirobira, Denis Efimov, Alexandre L Fradkov. On robust stability of multistable passive systems. ECC 2019 - 17th European Control Conference, Jun 2019, Naples, Italy. hal-02418552

\section{HAL Id: hal-02418552 \\ https://hal.inria.fr/hal-02418552}

Submitted on 18 Dec 2019

HAL is a multi-disciplinary open access archive for the deposit and dissemination of scientific research documents, whether they are published or not. The documents may come from teaching and research institutions in France or abroad, or from public or private research centers.
L'archive ouverte pluridisciplinaire HAL, est destinée au dépôt et à la diffusion de documents scientifiques de niveau recherche, publiés ou non, émanant des établissements d'enseignement et de recherche français ou étrangers, des laboratoires publics ou privés. 


\title{
On robust stability of multistable passive systems
}

\author{
N. de Figueiredo Barroso, R. Ushirobira, D. Efimov, A. L. Fradkov
}

\begin{abstract}
The problem of stability robustness with respect to external inputs is analyzed for passive and strict passive systems, whose storage and supply rate functions are sign-definite with respect to a set containing all compact and globally attracting invariant subsets of the unforced system. The results are obtained using the framework of inputto-state stability and integral input-to-state stability for multistable systems. Several conditions are proposed and the cases when an output stabilizing feedback is needed are highlighted. An application of the obtained conditions is illustrated on the model of multispecies populations.
\end{abstract}

\section{INTRODUCTION}

The analysis of stability and robustness properties of multistable systems has become more and more appealing from the perspective of systems and control theory due to its importance for several scientific disciplines ranging from mechanics and electronics [20], [11] to biology [29], [22], [27] and neuroscience [28]. Multistable systems include bistable systems (with at least two stable equilibria) [37], [5], almost globally stable systems (with only one attracting invariant set) [1], and nonlinear systems with generic invariant sets [3], [7], [12], [13], [19], [30], [35], [36].

The multistability phenomenon arises when it is necessary to analyze the global behavior of complex nonlinear dynamics, taking into account all its possible final states and motions. Recently, a new approach on multistability analysis was presented in [9] for autonomous systems. In that work, it was proposed a global asymptotic stability notion as well as the necessary and sufficient Lyapunov characterization for multistable systems, having as the object of investigation all compact invariant solutions of the system (including locally stable and unstable ones), extending the classical Lyapunov stability notion for systems with multiple invariant sets. The global nature of the stability and properties of Lyapunov functions have been also proved to be useful in robustness analysis with respect to external disturbances. In fact, it was made clear in [2] that the most natural way of investigating stability properties for multistable systems is to relax the Lyapunov stability requirement on its invariant sets under relatively mild additional assumptions on the decomposition of such sets. This intuitive path has led to a new line of research which starts from the characterizations of inputto-state stability (ISS) for this class of systems in terms of usual Lyapunov dissipation inequalities, generalizing the classical ISS theory [33], [34], [6], [2], [15]. Formulated within the framework introduced in [2], the notion of detectability or output-to-state

Nelson de Figueiredo Barroso, Rosane Ushirobira and Denis Efimov are with Inria, Univ. Lille, CNRS, UMR 9189 - CRIStAL, F-59000 Lille, France.

Alexander L. Fradkov is with Institute for Problems of Mechanical Engineering RAS, Saint Petersburg, Russia.

Denis Efimov is with Department of Control Systems and Informatics, University ITMO, 197101 Saint Petersburg, Russia.

The project is supported by the ANR project WaQMoS (ANR $15 \mathrm{CE}$ 04 0002). It is also partially supported by the Government of Russian Federation (Grant 08-08) and the Ministry of Education and Science of Russian Federation (Project 14.Z50.31.0031).

Nelson de Figueiredo Barroso is partly supported by the regional council of Hauts-de-France. stability (OSS) was generalized in [16]. Next, integral input-tostate stability (iISS) characterization, which is weaker than the classical one given in [32], [23], [4], was extended in [17] for systems with multiple invariant sets. Specifically, it was introduced a notion of iISS as the conjunction of global attractiveness with zero disturbances (0-GATT) and the uniform bounded energy boundedstate properties (UBEBS) providing again equivalent characterizations in terms of Lyapunov/LaSalle-like dissipation inequalities. Further investigations along the lines of multistable systems have addressed applications of such concepts to the analysis and synthesis on specific problems such as stability of nonlinear cascades and feedback interconnections [14] and periodic systems [11].

To contribute in this direction, we are interested in the analysis of passive systems. The class of passive dynamics is very large and omnipresent in mechanics, electric circuits and systems biology [24], [26], [18]. The problem of passivity and input-to-state stability of nonlinear systems with respect to an arbitrary compact and connected invariant set was treated in [8], [10] where the iISS (ISS) stabilizability property by output feedback for passive and strict passive system was considered. In the present work we are interested in an extension of such a global robustness analysis for passive systems in a context of multiple invariant sets (compact and maybe disconnected).

The outline of this work is as follows. The main definitions and problem statement are given in Section II, while the obtained results are presented in Section III. Section IV brings an example of application within the theoretical biology domain. Final remarks and discussion are summarized in Section V.

\section{Definitions and Problem Statement}

Let $\mathcal{M}$ be a $n$-dimensional smooth manifold without boundary, equipped with a metric $\delta: \mathcal{M} \times \mathcal{M} \rightarrow \mathbb{R}_{+}=\{s \in \mathbb{R}: s \geq 0\}$. Consider a nonlinear model of dynamical systems evolving on this manifold, of the following form:

$$
\begin{aligned}
\dot{x}(t) & =f(x(t), u(t)), \quad t \in \mathbb{R}_{+}, \\
y(t) & =h(x(t)),
\end{aligned}
$$

where $x(t) \in \mathcal{M}$ is the state vector, $u(t) \in \mathbf{U} \subseteq \mathbb{R}^{m}$ is the input vector, $u \in \mathcal{U}$, the set of admissible controls $\mathbb{R}_{+} \rightarrow \mathbf{U}$ (locally essentially bounded and measurable signals), and $y(t) \in \mathcal{Y} \subseteq \mathbb{R}^{p}$ is the output vector. Let $f: \mathcal{M} \times \mathbf{U} \rightarrow T_{x} \mathcal{M}$ be locally Lipschitz continuous on $\mathcal{M}$ (here $T_{x} \mathcal{M}$ denotes the tangent space of $\mathcal{M}$ ), and assume that $h: \mathcal{M} \rightarrow \mathbb{R}^{p}$ is continuously differentiable, $h(0)=0$ and $f(0,0)=0$.

Denote by $x\left(t, x_{0} ; u\right)$ the uniquely defined solution of (1) at time $t \geq 0$ fulfilling $x(0)=x_{0}$ under the input $u \in \mathcal{U}$. For the unperturbed system, i.e. the system (1) with $u \equiv 0$, we have:

$$
\dot{x}(t)=f(x(t), 0), \quad t \geq 0,
$$

we say that $S \subset \mathcal{M}$ is invariant if for all $x_{0} \in S, x\left(t, x_{0} ; 0\right) \in S$ for all $t \in \mathbb{R}$. For a set $S \subset \mathcal{M}$ and any $x \in \mathcal{M}$ define the corresponding distance as

$$
|x|_{S}=\inf _{a \in S} \delta(x, a),
$$


with a convention that for a point $x_{\text {or }} \in \mathcal{M}$, selected as the origin on $\mathcal{M},|x|=|x|_{\left\{x_{\text {or }}\right\}}$ can be considered as a norm. For a measurable function $g: \mathbb{R}_{+} \rightarrow \mathbb{R}^{m}$, define its $L_{\infty}$-norm as

$$
\|g\|_{\infty}=\operatorname{ess} \sup _{t \geq 0}|g(t)| \text {. }
$$

\section{A. Decomposition of a compact invariant set}

Let $\Lambda \subset \mathcal{M}$ be a compact invariant set for the unperturbed system (3). In order to characterize the evolution of this system along $\mathcal{M}$ it is useful to decompose $\Lambda$ and explicitly denote the existence of solutions traveling between different components of its decomposition.

Definition 1. [25] A decomposition of $\Lambda$ is a finite, disjoint family of compact invariant sets $\Lambda_{1}, \cdots, \Lambda_{k}$ such that

$$
\Lambda=\bigcup_{i=1}^{k} \Lambda_{i} .
$$

For an invariant set $\Lambda$, its attracting and repulsing subsets can be defined, respectively, as follows:

$$
\begin{aligned}
& \mathfrak{A}(\Lambda)=\left\{x_{0} \in \mathcal{M}:\left|x\left(t, x_{0} ; 0\right)\right|_{\Lambda} \rightarrow 0 \text { as } t \rightarrow+\infty\right\}, \\
& \mathfrak{R}(\Lambda)=\left\{x_{0} \in \mathcal{M}:\left|x\left(t, x_{0} ; 0\right)\right|_{\Lambda} \rightarrow 0 \text { as } t \rightarrow-\infty\right\} .
\end{aligned}
$$

Based on it, we can define a relation between two invariant sets $\mathcal{W} \subset \mathcal{M}$ and $\mathcal{D} \subset \mathcal{M}$ by $\mathcal{W} \prec \mathcal{D}$ if $\mathfrak{A}(\mathcal{W}) \cap \mathfrak{R}(\mathcal{D}) \neq \varnothing$. This relation implies that there is a solution connecting the set $\mathcal{D}$ with the set $\mathcal{W}$. A collection of $r$ disjoint sets that can be reached from one to another in a loop by a suitable concatenation of systems solutions is called $r$-cycle.

Definition 2. [25] Let $\Lambda_{1}, \cdots, \Lambda_{k}$ be a decomposition of $\Lambda$, then 1) An $r$-cycle $(r \geq 2)$ is an ordered $r$-tuple of distinct indices $i_{1}, \cdots, i_{r}$ such that $\Lambda_{i_{1}} \prec \cdots \prec \Lambda_{i_{r}} \prec \Lambda_{i_{1}}$.

2) A 1-cycle is an index $i$ such that $\left[\mathfrak{R}\left(\Lambda_{i}\right) \cap \mathfrak{A}\left(\Lambda_{i}\right)\right] \backslash \Lambda_{i} \neq \varnothing$.

3) A filtration ordering is a numbering of the $\Lambda_{i}$ so that $\Lambda_{i} \prec$ $\Lambda_{j} \Rightarrow i \leq j$.

However, what qualifies a decomposition for its treatment by means of Lyapunov-like analytical tools is the absence of cycles. Therefore, in the sequel we will consider the following assumption:

Assumption 1. [2] The compact invariant set $\mathcal{W}$, containing all $\alpha$ and $\omega$-limit sets of the unperturbed system $\dot{x}=f(x, 0)$, admits a finite decomposition without cycles: $\mathcal{W}=\bigcup_{i=1}^{k} \mathcal{W}_{i}$ for some nonempty disjoint compact sets $\mathcal{W}_{i}$, which form a filtration ordering of $\mathcal{W}$, as detailed in Definition 2.

Notice that in most examples one might be able to choose $\mathcal{W}=\mathcal{A} \cup \mathcal{R} \cup \mathcal{H}$, where the set $\mathcal{A}$ is composed by locally asymptotically stable invariant sets, the set $\mathcal{R}$ contains locally antistable invariant sets and $\mathcal{H}=\mathcal{H}^{+} \cap \mathcal{H}^{-}$is a hyperbolic invariant set, where $\mathcal{H}^{+}$and $\mathcal{H}^{-}$constitute, respectively, stable and unstable invariant submanifold for $\mathcal{H}$. Some of these sets may be empty [9], [2].

\section{B. Robust stability notions for decomposable $\mathcal{W}$}

In this subsection, we list several ISS and iISS stability properties for system (1) with respect to $\mathcal{W}$ satisfying Assumption 1. Most of the following properties are direct extensions of the classical ISS and iISS notions introduced in [33], [34], [23], [4].

The definitions of function classes $\mathcal{K}$ and $\mathcal{K}_{\infty}$ can be found in [6]. A function $V: \mathcal{M} \rightarrow \mathbb{R}_{+}$is called positive definite if it vanishes only at the origin, and proper unbounded if $V(x) \rightarrow+\infty$ for $|x|_{\mathcal{W}} \rightarrow+\infty$. The Lie derivative of such a continuously differentiable function $V$ in the line of a vector field $f: \mathcal{M} \rightarrow \mathbb{R}^{n}$ is denoted by:

$$
D V(x) f(x, u)=\frac{\partial V(x)}{\partial x} f(x, u) .
$$

1) Input-to-state stability:

Definition 3. [2] The system (1) has the practical asymptotic gain (pAG) property if there exist $\eta \in \mathcal{K}_{\infty}$ and $q \geq 0$ such that for all $x_{0} \in \mathcal{M}$ and all $u \in \mathcal{U}$ the solutions are defined for all $t \geq 0$ and the following holds:

$$
\limsup _{t \rightarrow+\infty}\left|x\left(t, x_{0}, u\right)\right|_{\mathcal{W}} \leq \eta\left(\|u\|_{\infty}\right)+q
$$

If $q=0$, then we say that the asymptotic gain (AG) property holds.

Definition 4. [2] The system (1) has the limit property (LIM) with respect to $\mathcal{W}$ if there exists $\mu \in \mathcal{K}_{\infty}$ such that for all $x_{0} \in \mathcal{M}$ and all $u \in \mathcal{U}$ the solutions are defined for all $t \geq 0$ and the following holds:

$$
\inf _{t \geq 0}\left|x\left(t, x_{0}, u\right)\right|_{\mathcal{W}} \leq \mu\left(\|u\|_{\infty}\right) .
$$

Definition 5. [2] The system (1) has the practical global stability (pGS) property with respect to $\mathcal{W}$ if there exists $\beta \in \mathcal{K}_{\infty}$ and $c \geq 0$ such that for all $x_{0} \in \mathcal{M}$ and all $u \in \mathcal{U}$ the following holds for all $t \geq 0$ :

$$
\left|x\left(t, x_{0} ; u\right)\right|_{\mathcal{W}} \leq \beta\left(\max \left\{\left|x_{0}\right|_{\mathcal{W}}+c,\|u\|_{\infty}\right\}\right)
$$

(which is equivalent to

$$
\left|x\left(t, x_{0} ; u\right)\right|_{\mathcal{W}} \leq \tilde{\beta}\left(\max \left\{\left|x_{0}\right|_{\mathcal{W}},\|u\|_{\infty}\right\}\right)+q
$$

for some $\tilde{\beta} \in \mathcal{K}_{\infty}$ and $q \geq 0$ ).

Definition 6. [2] A $\mathcal{C}^{1}$ function $V: \mathcal{M} \rightarrow \mathbb{R}$ is a practical ISSLyapunov function for (1) if there exist $\mathcal{K}_{\infty}$ functions $\alpha_{1}, \alpha_{2}, \alpha_{3}$ and $\gamma$, and $q \geq 0, c \geq 0$ such that

$$
\alpha_{1}\left(|x|_{\mathcal{W}}\right) \leq V(x) \leq \alpha_{2}\left(|x|_{\mathcal{W}}\right)+c
$$

and the following dissipation inequality holds for all $(x, u) \in \mathcal{M} \times$ U:

$$
D V(x) f(x, u) \leq-\alpha_{3}\left(|x|_{\mathcal{W}}\right)+\gamma(|u|)+q .
$$

If (5) holds for $q=0$, then $V$ is said to be ISS-Lyapunov function.

The existence of $\alpha_{2}$ and $c$ follows, without any additional hypothesis, by standard continuity arguments. Under Assumption 1 , whenever the $\mathcal{W}_{i}$ are recurrent invariant sets of the unperturbed system, any Lyapunov function which is non-increasing along solutions of (3) also takes constant values on any $\mathcal{W}_{i}, 1 \leq i \leq k$, from the decomposition of $\mathcal{W}$.

Theorem 7. [2] Consider a nonlinear system (1) and let $\mathcal{W}$ be a compact invariant set containing all $\alpha$ - and $\omega$-limit sets of the system (3) as in Assumption 1. Then the following facts are equivalent:

1. The system enjoys the $A G$ property.

2. The system admits an ISS Lyapunov function.

3. The system admits an ISS Lyapunov function constant on invariant sets.

4. The system admits a practical ISS Lyapunov function.

5. The system enjoys the pAG property.

6. The system enjoys the LIM property and the pGS. 
The system as in (1) that satisfies these properties will be called ISS in the multistable sense with respect to the set $\mathcal{W}$ and input $u$.

\section{2) Integral input-to-state stability:}

Definition 8. [17] The system (1) has the uniform bounded-energy bounded-state (UBEBS) property if for some $\alpha, \gamma, \sigma \in \mathcal{K}_{\infty}$, and some positive constant $c$, the following estimate holds for all $t \geq 0$, all $x_{0} \in \mathcal{M}$ and all $u \in \mathcal{U}$ :

$$
\alpha\left(\left|x\left(t, x_{0} ; u\right)\right| \mathcal{W}\right) \leq \gamma\left(\left|x_{0}\right| \mathcal{W}\right)+\int_{0}^{t} \sigma(|u(\tau)|) d \tau+c .
$$

Definition 9. [17] The system (1) has the zero-global attraction (0GATT) property with respect to a compact invariant set $\mathcal{W}$, if every trajectory $x\left(t, x_{0} ; 0\right)$ of the unperturbed system (3) satisfies

$$
\lim _{t \rightarrow+\infty}\left|x\left(t, x_{0} ; 0\right)\right|_{\mathcal{W}}=0 .
$$

Definition 10. [17] The system (1) has the bounded-energy weakly converging state (BEWCS) property if there exists a function $\tilde{\sigma} \in$ $\mathcal{K}_{\infty}$ such that the following estimate holds for all $x_{0} \in \mathcal{M}$ and $u \in \mathcal{U}$ :

$$
\int_{0}^{+\infty} \tilde{\sigma}(|u(\tau)|) d \tau<+\infty \Rightarrow \liminf _{t \rightarrow+\infty}\left|x\left(t, x_{0} ; u\right)\right|_{\mathcal{W}}=0 .
$$

Definition 11. [17] The system (1) has the bounded-energy strongly converging state (BESCS) property if there exists a function $\tilde{\sigma} \in$ $\mathcal{K}_{\infty}$ such that the following estimate holds for all $x_{0} \in \mathcal{M}$, and $u \in \mathcal{U}$ :

$$
\int_{0}^{+\infty} \tilde{\sigma}(|u(\tau)|) d \tau<+\infty \Rightarrow \lim _{t \rightarrow+\infty}\left|x\left(t, x_{0} ; u\right)\right|_{\mathcal{W}}=0 .
$$

Definition 12. [17] A $\mathcal{C}^{1}$ function $V: \mathcal{M} \rightarrow \mathbb{R}$ is called iISS-Lyapunov function for the system (1) if there exist functions $\alpha_{1}, \alpha_{2}, \sigma \in \mathcal{K}_{\infty}, c \geq 0$, and a continuous positive definite function $\alpha_{3}: \mathbb{R}_{+} \rightarrow \mathbb{R}_{+}$such that the relations (4) are satisfied for all $x \in \mathcal{M}$, and the following dissipation inequality holds for all $(x, u) \in \mathcal{M} \times \mathbf{U}:$

$$
D V(x) f(x, u) \leq-\alpha_{3}\left(|x|_{\mathcal{W}}\right)+\sigma(|u|) .
$$

Definition 13. [17] The system (1), (2) has the smooth dissipativity property if there exist $\mathcal{C}^{1}$ function $V: \mathcal{M} \rightarrow \mathbb{R}$, functions $\alpha_{1}, \alpha_{2}, \sigma \in \mathcal{K}_{\infty}$, a continuous positive definite function $\alpha_{4}$, and a continuous output map $h: \mathcal{M} \rightarrow \mathbb{R}^{p}$ with

$$
|x|_{\mathcal{W}}=0 \Rightarrow h(x)=0, \quad \forall x \in \mathcal{M},
$$

such that (4) is satisfied for all $x \in \mathcal{M}$, and the following dissipation inequality holds for all $(x, u) \in \mathcal{M} \times \mathbf{U}$ :

$$
D V(x) f(x, u) \leq-\alpha_{4}(|h(x)|)+\sigma(|u|) .
$$

Definition 14. [17] The system (1), (2) has the weak zerodetectability property if the following relation holds:

$h\left(x\left(t, x_{0} ; 0\right)\right) \equiv 0, \quad \forall t \geq 0 \Rightarrow\left|x\left(t, x_{0} ; 0\right)\right|_{\mathcal{W}} \rightarrow 0 \quad$ as $\quad t \rightarrow+\infty$.

Theorem 15. [17] Consider a nonlinear system (1) and let $\mathcal{W}$ be a compact invariant set containing all $\alpha$-and $\omega$-limit sets of the system (3) as in Assumption 1. Then the following facts are equivalent:

1. 0-GATT and UBEBS or BEWCS properties.

2. Existence of a smooth iISS Lyapunov function $V$ such that $D V(x)=0$ for all $x \in \mathcal{W}$.

3. Existence of a $\mathcal{C}^{1}$ iISS Lyapunov function $V$.
4. Existence of an output function that makes the system smoothly dissipative and weakly zero detectable.

\section{BESCS property.}

The system as in (1) that satisfies these properties will be called iISS in the multistable sense with respect to the set $\mathcal{W}$ and input $u$.

Lemma 16. [17] Let system (1) be 0-GATT. Then, there exist a smooth function $U: \mathcal{M} \rightarrow \mathbb{R}$, functions $\nu_{1}, \nu_{2}, \delta \in \mathcal{K}$, a continuous positive definite function $\varpi: \mathbb{R}_{+} \rightarrow \mathbb{R}_{+}$, and a positive constant $c$ such that:

$$
\begin{gathered}
\nu_{1}\left(|x|_{\mathcal{W}}\right) \leq U(x) \leq \nu_{2}\left(|x|_{\mathcal{W}}+c\right), \\
D U(x) f(x, u) \leq-\varpi\left(|x|_{\mathcal{W}}\right)+\delta(|u|) .
\end{gathered}
$$

for all $x \in \mathcal{M}$ and $u \in \mathbf{U}$. Moreover, $D U(x)=0$ for all $x \in \mathcal{W}$.

Note that if the function $U$ in Lemma 16 were proper, i.e. $\nu_{1} \in \mathcal{K}_{\infty}$, then it would qualify $U$ as an iISS-Lyapunov function. Unfortunately, this is not necessarily the case, therefore $U$ needs to be used in addition to a proper function $V$ to obtain a new iISSLyapunov function such that the iISS property holds.

\section{Passive and strict passive systems}

Definition 17. [21] The system (1), (2) is passive with a continuous function $V: \mathcal{M} \rightarrow \mathbb{R}_{+}$, if for all $x_{0} \in \mathcal{M}, u \in \mathcal{U}, t \geq 0$ the following inequality is satisfied:

$V\left(x\left(t, x_{0}, u\right)\right) \leq V\left(x_{0}\right)+\int_{0}^{t} \varpi\left(x\left(\tau, x_{0} ; u\right), u(\tau), y\left(\tau, x_{0} ; u\right)\right) d \tau$,

$$
\varpi(x, u, y)=y^{T} u-\beta(x),
$$

where $\beta: \mathcal{M} \rightarrow \mathbb{R}_{+}$is a continuous function. Then $\varpi$ and $V$ are called, respectively, supply rate and storage functions.

A system is called passive with a certain rate of dissipation $\beta$ if in (8) the equality sign can be used. It is called passive without losses if in (8) it is possible to use the equality sign and $\beta(\cdot) \equiv 0$. Finally, if in (9) $\beta$ is a positive definite function, then the system is called strictly passive.

If the storage function $V$ is continuously differentiable, then the inequality on the trajectories (8) gives a simpler form:

$$
D V(x) f(x, u) \leq \varpi(x, u, y)
$$

that has to be verified for all $x \in \mathcal{N}, u \in \mathbf{U}$ and $y \in \mathcal{Y}$.

A system that admits the passive property with a proper storage function $V$ is Lyapunov stable for $u(t) \equiv 0, t \geq 0$, and the strictly passive system in such a case is asymptotically Lyapunov stable with a Lyapunov function $V$ (these facts can be proven using LaSalle arguments since positive definiteness of the storage function $V$ has not been claimed).

In this paper, we will also deal with a subclass of nonlinear dynamical system (1), (2) affine in the input of the following form:

$$
\begin{aligned}
\dot{x}(t) & =F(x(t))+G(x(t)) u(t), \quad t \in \mathbb{R}_{+}, \\
y(t) & =h(x(t)),
\end{aligned}
$$

where as before $x(t) \in \mathcal{M}$ is the state vector, $u(t) \in \mathbf{U} \subseteq \mathbb{R}^{m}$ is the input vector, $u \in \mathcal{U}$, and $y(t) \in \mathcal{Y} \subseteq \mathbb{R}^{p}$ is the output vector. For this system $f(x, u)=F(x)+G(x) u$, where $F: \mathcal{M} \rightarrow$ $\mathbb{R}^{n}$ and the columns of the matrix function $G: \mathcal{M} \rightarrow \mathbb{R}^{n \times m}$ are assumed to be locally Lipschitz continuous on $\mathcal{M}, h: \mathcal{M} \rightarrow \mathbb{R}^{p}$ is continuously differentiable, and $F(0)=h(0)=0$. The KalmanYakubovich-Popov Lemma claims that for the system (10), (11) 
the output function for passive and strict passive systems with a differentiable storage function can be defined in the following way:

$$
h(x)=(D V(x) G(x))^{T}=\left(\frac{\partial V}{\partial x} G(x)\right)^{T} .
$$

\section{Problem statement}

Passivity and strict passivity properties of a dynamical system mean that the system is, respectively, Lyapunov stable and asymptotically Lyapunov stable for zero inputs. However, in the general case these properties are not robust with respect to input perturbations and an arbitrary small input signal can initiate unstable processes in the system [8], [10]. Therefore, the task of iISS (ISS) stabilization of such a kind of systems is of great interest.

Definition 18. [31] The system (10), (11) is referred to as iISS (ISS) stabilizable if there exists for it a control law

$$
u(t)=k(x(t))+v(t),
$$

where $k: \mathcal{M} \rightarrow \mathbf{U}$ is a Lipschitz continuous function and $v(t) \in \mathbf{U}$, $v \in \mathcal{U}$ is a new input vector, rendering the closed-loop system to be iISS (ISS) with respect to $v$.

Thus, the problem studied in this work can be formally written introducing the following hypothesis:

Assumption 2. A passive or strict passive system described by the affine nonlinear model (10), (11) has a decomposable compact invariant set $\mathcal{W}$ as in Assumption 1 and its storage function $V$ : $\mathcal{M} \rightarrow \mathbb{R}_{+}$is continuously differentiable and satisfies (4) for all $x \in \mathcal{M}$, while the supply rate has the form:

$$
\varpi(x, u, y)=y^{T} u-\beta\left(|x|_{\mathcal{W}}\right),
$$

where $\beta: \mathbb{R}_{+} \rightarrow \mathbb{R}_{+}$is a continuous function.

Problem. Under Assumption 2, find conditions of iISS or ISS stabilizability for (10), (11) (according to Definition 18).

As we can see, to choose a control law that provides the iISS (ISS) property for a strict passive system (10), (11) in the case of multiple invariant sets, we need to introduce some specifications on the storage function and the supply rate (these additional requirements do not contradict to Definition 17). Hence, the evolution of the states $x \in \mathcal{M}$ must be evaluated with respect to the invariant set $\mathcal{W} \subset \mathcal{M}$ (the functions $V$ and $\beta$ are defined with respect to $|x|_{\mathcal{W}}$ ), and we have to assume that this set is globally attractive for $u \equiv 0$. Another interesting point consists in distinguishing the situations where an additional feedback $k(x)$ is needed, or where we can select $k(\cdot)=0$.

\section{MAIN RESULTS}

In this section, we present several results about different conditions on robust stability and stabilizability of passive systems. We omit the proofs for reasons of space.

Lemma 19. Let a strict passive system (10), (11) admit Assumption 2. Then, the control law

$$
u=-\varphi(y)+v
$$

with a Lipschitz continuous function $\varphi: \mathcal{Y} \rightarrow \mathbf{U}$ satisfying $y^{T} \varphi(y)>0$ for all $y \neq 0$ and $v \in \mathcal{U}$, ensures iISS property for the system with respect to $\mathcal{W}$ and the disturbance input $v$, provided that one of the following conditions holds:

$$
\begin{gathered}
\lim _{|x| \mathcal{W} \rightarrow+\infty} \frac{|h(x)|}{V(x)}<+\infty, \\
y^{T} \varphi(y) \geq \varepsilon|y|^{2}, \quad \forall y \in \mathcal{Y}, \varepsilon>0 .
\end{gathered}
$$

If the condition (15) is satisfied and the function $\beta \in \mathcal{K}_{\infty}$ or $|h(x)| \geq \varrho\left(|x|_{\mathcal{W}}\right)$ for all $x \in \mathcal{M}$, then the control (13) guarantees ISS property with respect for the set $\mathcal{W}$ and the disturbance input $v$, and $V$ is an ISS-Lyapunov function.

Lemma 19 establishes the connection between the form of function $\beta$ and robustness property of the system with respect to $L_{\infty}$ or $L_{2}$ inputs. An important consequence of this lemma consists in the ability of a strict passive system to become robust with respect to additive perturbation in the input channel under any output feedback with an arbitrary small gain $\varepsilon$ [8], [10], [27].

Lemma 20. Let a strict passive system (10), (11) admit Assumption 2 and the following inequality hold for all $x \in \mathcal{M}$ :

$$
|D V(x) G(x)| \leq b(V(x))
$$

where $b: \mathbb{R}_{+} \rightarrow \mathbb{R}_{+}$is a function satisfying the integral constraint:

$$
\int_{0}^{+\infty} \frac{d r}{1+b(r)}=+\infty .
$$

Then the system is iISS with respect to $\mathcal{W}$ and the input $u$.

As we can conclude from Theorem 15, the iISS property is rather weak, it lies in the range between 0-GATT and BESCS properties. The conditions of the above lemmas expose the technical details of this range for a strict passive system. Now let us consider the same issues for passive systems only.

Lemma 21. Let a passive system (10), (11) admit Assumption 2. If the system possesses the weak zero-detectability property and the conditions (14) and

$$
\frac{\varphi(|h(x)|)}{V(x)}|h(x)| \geq \kappa(|h(x)|), \quad \forall x \in \mathcal{M},
$$

with a positive definite continuous function $\kappa: \mathbb{R}_{+} \rightarrow \mathbb{R}_{+}$hold, then the control law (13) provides the iISS property for this system with respect to $\mathcal{W}$ and the input $v$.

Note that Assumption 2 implies 0-GATT property (it refers to Assumption 1, where it is stated that $\mathcal{W}$ contains all $\alpha$ - and $\omega$-limit sets of the unperturbed system $\dot{x}=f(x, 0)$, thus it is the global attractor in the system for $u=0$ ).

Lemma 22. Let a passive system (10), (11) admit Assumption 2. If the control (13) is applied under (15) and an additional restriction:

$$
\delta(2|\varphi(y)|) \leq \epsilon|y|^{2}, \quad \forall y \in \mathcal{Y}, \epsilon>0,
$$

where $\delta \in \mathcal{K}$ is given in Lemma 16, then the control law (13) provides the iISS property for this system with respect to $\mathcal{W}$ and the input $v$.

\section{EXAMPLE}

To exemplify the ISS (iISS) theory application, we will consider the famous $N$-species Lotka-Volterra (predator-pray) model [29] in its controlled version introduced in [27]. In this model it is supposed that for the population of $N>1$ species, the birth rate of the species $x_{\ell} \in \mathbb{R}_{+}, \ell=M+1, \ldots, N$ can be controlled with the index 
$M \in[0, N]$. Then the interaction between the species is described by the following system of differential equations:

$$
\begin{aligned}
& \dot{x}_{i}(t)=x_{i}\left(k_{i}+\beta_{i}^{-1} \sum_{j=1}^{N} a_{i j} x_{j}(t)\right), \quad i=1,2, \ldots, M, \\
& \dot{x}_{\ell}(t)=x_{\ell}\left(k_{\ell}+\beta_{\ell}^{-1} \sum_{j=1}^{N} a_{\ell j} x_{j}(t)+u_{\ell}\right), \quad \ell=M+1, \ldots, N,
\end{aligned}
$$

where $u=\left[u_{M+1}, \ldots, u_{N}\right]^{T} \in \mathbb{R}^{N-M}$ is the control, $k_{i}\left(k_{\ell}\right)$ is the speed of the natural increase or death rate of the $i$-th $(\ell$-th) species in the absence of all others with the following convention: $k_{i}<0$ $\left(k_{\ell}<0\right)$, if the $i$-th $(\ell$-th) species lives at the expense of others and $k_{i}>0\left(k_{\ell}>0\right)$ otherwise. The parameter $\beta_{i}>0\left(\beta_{\ell}>0\right)$ reflects the fact that the appearance of a predator is usually connected with the vanishing of one or more preys. The quantities $a_{i j}, i \neq j\left(a_{\ell j}\right.$, $\ell \neq j$ ) evaluate the type and the intensity of the interaction between $i$-th ( $\ell$-th) and $j$-th species and form an asymmetric matrix.

Assume that there exists at least one positive equilibrium of (17) for some values of the system parameters:

$$
n=\left(n_{1}, n_{2}, \cdots, n_{N}\right), \quad n_{i}>0 \quad i=1, \ldots, N,
$$

and consider an auxiliary function $W$ :

$$
W(x)=\sum_{i=1}^{N} \beta_{i} n_{i}\left(\frac{x_{i}}{n_{i}}-\ln \left(\frac{x_{i}}{n_{i}}\right)\right) .
$$

If the condition (18) holds, then $W(x)$ is constant along the trajectories of (17) for $u_{\ell}=0$ with $\ell=M+1, \ldots, N$. Hence, by introducing the control goal

$$
W(x(t)) \rightarrow W^{*}, \quad t \rightarrow \infty,
$$

a desired amplitude of oscillations can be achieved. Note that if $W^{*}=W(n)=\min _{x \in M} W(x)$, the goal (19) means achievement of the equilibrium $x=n$.

Then, the problem is to find a control function $u(t)$ stabilizing the desired level $W^{*}$ of the function $W$ and hence providing an oscillatory property to the system with the needed amplitude of oscillations. To this end it is necessary to introduce another auxiliary function

$$
Q(x)=\frac{1}{2}\left(W(x)-W^{*}\right)^{2},
$$

that can be considered as a storage function for (17).

Following the SG method the control action is chosen as

$$
u_{\ell}(t)=-\gamma_{\ell}\left(W(x)-W^{*}\right)\left(x_{\ell}(t)-n_{\ell}\right)+v_{\ell}
$$

for some $\gamma_{\ell}>0$ and all $\ell=M+1, \ldots, N$, where $v=$ $\left[v_{M+1}, \ldots, v_{N}\right]^{T} \in \mathbb{R}^{N-M}$ is a disturbance input (essentially bounded function of time) added to the system in order to investigate the ISS (iISS) stabilizability property and to represent the model uncertainty. Then, by proceeding with the derivative of $Q(x)$ along the system (17) and substituting (20) we achieve:

$$
\begin{aligned}
\dot{Q}(x, u) \leq- & \gamma\left|\left(W(x)-W^{*}\right) \sum_{\ell=M+1}^{N}\left(x_{\ell}(t)-n_{\ell}\right)\right|^{2} \\
& +\left|\left(W(x)-W^{*}\right) \sum_{\ell=M+1}^{N}\left(x_{\ell}(t)-n_{\ell}\right)\right|\left|v_{\ell}\right|,
\end{aligned}
$$

where $\gamma=\min _{\ell=M+1, \ldots, N}\left\{\gamma_{\ell}\right\}$. Therefore, the set of all invariant solutions of the system for $v=0$ is given by $\mathcal{W}=\{n\} \cup \Gamma$, where $\Gamma=\left\{x: W(x)=W^{*}\right\}$.
Finally, we have:

$$
\dot{Q}(x, u) \leq-\gamma|y|^{2}+|y||v| \leq-0.5 \varepsilon|y|^{2}+0.5 \varepsilon^{-1}|v|^{2}
$$

for some $\varepsilon>0$. Using the same arguments as in Lemma 22, it is easy to see that the smooth dissipativity property holds for this system. Also, one can show that for $M=0$ the equation (21) can be written as an ISS-Lypunov function in the form:

$$
\dot{Q}(x, u) \leq-\alpha_{3}\left(|x|_{\mathcal{W}}\right)+0.5 \varepsilon^{-1}|v|^{2} .
$$

where $\alpha_{3}$ is a $\mathcal{K}_{\infty}$ function rendering to the system the ISS property.

Now, we will illustrate by means of a simple numerical experiment the convergence of the system trajectories to a set $\mathcal{W}$. For that, let us consider a system with one predator and one prey:

$$
\begin{aligned}
& \dot{x_{1}}=k_{1} x_{1}+\beta_{1}^{-1} a_{12} x_{1} x_{2}, \\
& \dot{x_{2}}=k_{2} x_{2}+\beta_{2}^{-1} a_{21} x_{1} x_{2}+x_{2} u_{2},
\end{aligned}
$$

where $x_{1} \in \mathbb{R}_{+}$and $x_{2} \in \mathbb{R}_{+}$represent, respectively, the predator and the prey populations; $k_{1}=-9, k_{2}=7, \beta_{1}=5, \beta_{2}=4$, $a_{12}=2$, and $a_{21}=-a_{12}$ [27]. The equilibrium for the system (22) for these parameters is $n_{1}=14$ and $n_{2}=22.5$. Therefore, the quantity $W(n) \cong 160$. For simulations, we choose as the initial condition the point $x(0)=\left[\begin{array}{ll}15 & 25\end{array}\right]^{T}$, which results in $W(x(0)) \cong$ 160.69. We will choose $W^{*}=175$, and apply the control law (20) without and with the disturbance input $v$. In particular, the control action (20) for our example is given by:

$$
u_{2}(t)=-\gamma_{2}\left(W(x)-W^{*}\right)\left(x_{2}(t)-n_{2}\right)+v_{2},
$$

where it has been selected for simulations $\gamma_{2}=0.01$ and $v_{2}=$ $\sin (20 t)$.

The phase portrait for the controlled system (22) is shown in Fig. 1. As we can conclude, for the case without disturbance the trajectories of the closed-loop system converge to the desired limit cycle, indirectly measured by $W(x)$. For the case where there exists a disturbance, it is possible to see that the boundedness is keeping with some deviations of trajectories around the limit cycle without achieving zero for none of the species. The behavior of $W(x)$ for the controlled system (22) without and with disturbance input is shown in Fig. 2. This oscillation can be conveniently decreased by increasing the gain $\gamma_{2}$. Therefore, these results of numerical experiments confirm the theoretical findings of the paper.

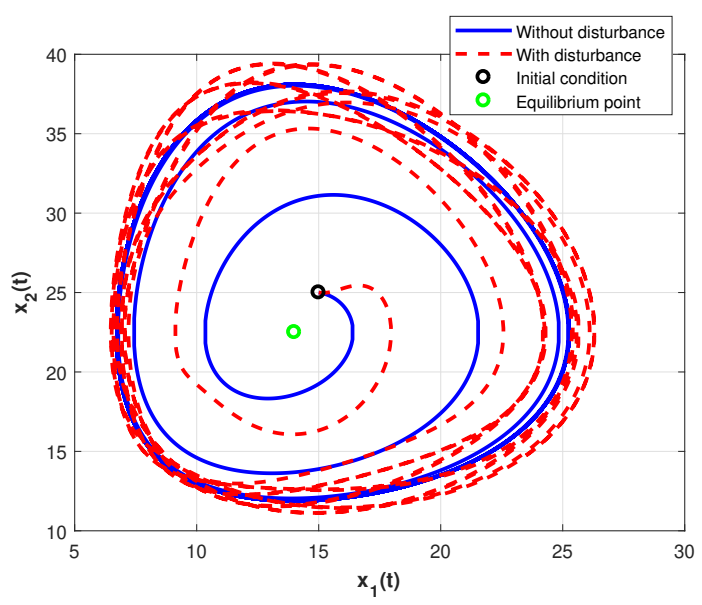

Figure 1: Phase portrait for the controlled system (22) without and with disturbance input, and $W^{*}=175$ 


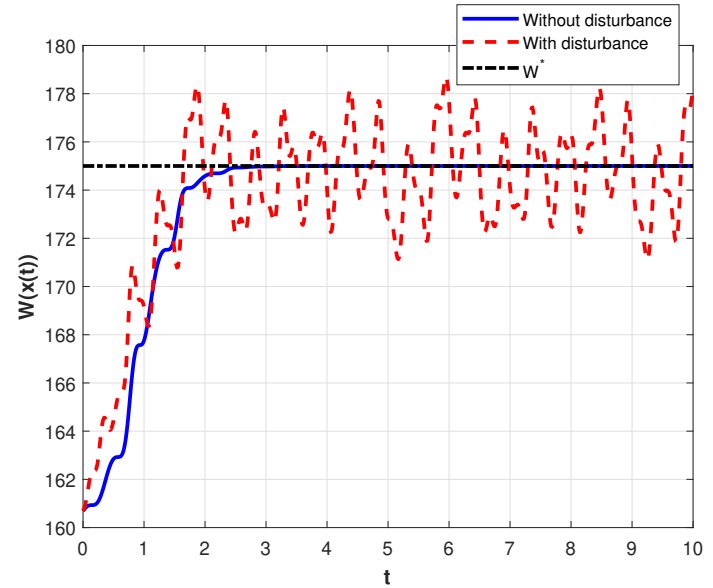

Figure 2: Behavior of $W(x)$ for the controlled system (22) without and with disturbance input, and $W^{*}=175$

\section{CONCLUSION}

The problems of ISS and iISS of passive and strict passive systems have been studied in the context of multistable dynamics and correspondingly defined storage and supply functions. Several conditions are proposed, which distinguish the cases when an additional output feedback is necessary or not to guarantee robustness against additional perturbations in the input channel. The obtained results are illustrated by the model of multispecies population dynamics, and in particular it is shown that if all species are controlled, then the ISS property can be recovered, but if a part of them can be regulated directly, then only the iISS property can be provided. For future research, the developments of general design methods for ISS or iISS stabilization of multistable systems can be considered.

\section{REFERENCES}

[1] D. Angeli. An almost global notion of input-to-state stability. IEEE Trans. Automatic Control, 49:866-874, 2004.

[2] D. Angeli and D. Efimov. Characterization of input-to-state stability for systems with multiple invariant sets. IEEE Transactions on Automatic Control, 60:3242-3256, 2015.

[3] D. Angeli, J.E. Ferrell, and E.D. Sontag. Detection of multistability, bifurcations and hysteresis in a large class of biological positivefeedback systems. Proc. Natl. Acad. Sci. USA, 101:1822-1827, 2004.

[4] D. Angeli, E. D. Sontag, and Y. Wang. A characterization of integral input-to-state stability. IEEE Transactions on Automatic Control, 45:1082-1097, 2000.

[5] M. Chaves, T. Eissing, and F. Allgöwer. Bistable biological systems: A characterization through local compact input-to-state stability. IEEE Trans. Automatic Control, 45:87-100, 2008.

[6] S. N. Dashkovskiy, D. Efimov, and E. D. Sontag. Input to state stability and allied system properties. Automation and Remote Control, 72:1579-1614, 2011.

[7] D. Dudkowski, S. Jafari, T. Kapitaniak, N. V. Kuznetsov, G. A. Leonov, and A. Prasad. Hidden attractors in dynamical systems. Physics Reports, 637:1-50, 2016.

[8] D. Efimov. Passivity and input-to-state stability of nonlinear systems. IFAC Proceedings Volumes, 39:285-290, 2006.

[9] D. Efimov. Global Lyapunov analysis of multistable nonlinear systems. SIAM Journal on Control and Optimization, 50(5):3132-3154, 2012.

[10] D. Efimov and A.L. Fradkov. Adaptive input-to-output stabilization of nonlinear systems. International Journal of Adaptive Control and Signal Processing, 22:949-967, 2008.

[11] D. Efimov, J. Schiffer, N. Barabanov, and R. Ortega. A relaxed characterization of iss for periodic systems with multiple invariant sets. European Journal of Control, 37:1-7, 2017.
[12] G. Enciso and E.D. Sontag. Monotone systems under positive feedback: multistability and a reduction theorem. Systems \& Control Lett., 54:159-168, 2005.

[13] F. Forni and R. Sepulchre. Differential analysis of nonlinear systems: Revisiting the pendulum example. In Proc. 53rd IEEE Conference on Decision and Control, pages 3848-3859, Los Angeles, US, 2014.

[14] P. Forni and D. Angeli. Input-to-state-stability for cascade systems with multiple invariant sets. Systems \& Control Letters, 98:97-110, 2016.

[15] P. Forni and D. Angeli. The iss approach to the stability and robustness properties of nonautonomous systems with decomposable invariant sets: An overview. European Journal of Control, 30:50-60, 2016.

[16] P. Forni and D. Angeli. Output-to-state stability for systems on manifolds with multiple invariant sets. IEEE Conference on Decision and Control, 55:453-458, 2016.

[17] P. Forni and D. Angeli. Characterization of integral input-to-state stability with multiple invariant sets. IEEE Transactions on Automatic Control, 62:3729-3743, 2017.

[18] A.L. Fradkov. Cybernetical Physics: From Control of Chaos to Quantum Control. Understanding Complex Systems. Springer-Verlag, London, 2007.

[19] J. Guckenheimer and P.J. Holmes. Nonlinear Oscillations, Dynamical Systems, and Bifurcations of Vector Fields, volume 42 of Applied Mathematical Sciences. Springer-Verlag, New York, 1983.

[20] C. Hayachi. Nonlinear oscillations in physical systems. McGraw-Hill Book Company, New York, 1964.

[21] D. Hill and P. Moylan. Dissipative dynamical systems: Basic inputoutput and state properties. Journal of the Franklin Institute, 309:327$357,1980$.

[22] M. Laurent and N. Kellershohn. Multistability: a major means of differentiation and evolution in biological systems. Trends Biochem. Sci., 24:418-422, 1999.

[23] D. Liberzon, E. D. Sontag, and Y. Wang. On integral-input-tostate stabilization. Proceedings of the American Control Conference, 3:1598-1602, 1999.

[24] H. Nijmeijer and A. J. van der Schaft. Nonlinear Dynamical Control Systems. Springer-Verlag, 1990.

[25] Z. Nitecki and M. Shub. Filtration, decompositions, explosions. American Journal of Mathematics, 97:1029-1047, 1975.

[26] R. Ortega, P.J. Loría, A. amd Nicklasson, and H.J. Sira-Ramirez. Passivity-based Control of Euler-Lagrange Systems: Mechanical, Electrical and Elctromechanical Aoolications. Springer-Verlag, London, 1998.

[27] I. Pchelkina and A. L. Fradkov. Control of oscillatory behavior of multispecies populations. Ecological Modelling, 227:1-6, 2012.

[28] A.N. Pisarchik and U. Feudel. Control of multistability. Physics Reports, 540:167-218, 2014.

[29] N. Rouche, P. Habets, and M. Laloy. Stability Theory by Liapunov's Direct Method. Springer-Verlag, Heidelberg, 1977.

[30] V.V. Rumyantsev and A.S. Oziraner. Stability and stabilization of motion with respect to part of variables. Nauka, Moscow, 1987. [in Russian].

[31] E. D. Sontag. Further facts about input-to-state stabilization. IEEE Transactions on Automatic Control, 35:473-476, 1990.

[32] E. D. Sontag. Coments on integral variants of iss. Systems \& Control Letters, 34:93-100, 1998

[33] E. D. Sontag and Y. Wang. On characterizations of the input-to-state stability property. Systems \& Control Letters, 24:351-359, 1995.

[34] E. D. Sontag and Y. Wang. New characterizations of the input-tostate stability. IEEE Transactions on Automatic Control, 41:1283-1294, 1996.

[35] G.-B. Stan and R. Sepulchre. Analysis of interconnected oscillators by dissipativity theory. IEEE Trans. Automatic Control, 52:256-270, 2007.

[36] V.I. Vorotnikov. Partial Stability and Control. Birkhauser, Boston, 1998.

[37] V.A. Yakubovich, G.A. Leonov, and A.Kh. Gelig. Stability of Stationary Sets in Control Systems with Discontinuous Nonlinearities. World Scientific, Singapore, 2004. [in Russian]. 